\title{
Energy drinks consumption among adolescent and its relation to obesity in the state of Qatar
}

\begin{abstract}
Introduction: The term obesity refers to having too much body fat, rather than being overweight due to the presence of high volumes of muscle mass, bone mass, or water. Obesity is a complex condition, with serious social and psychological dimensions, affecting virtually all ages and socioeconomic groups. Obesity associated with risks which increase incrementally in magnitude and severity as an individual's obesity level increases. Energy drinks are a large and growing beverage industry now marketed to children and adolescents for a variety of uses. Adolescent's consumption of energy drinks become common to a wide variety of inappropriate uses.
\end{abstract}

Objective: To examine patterns of adolescent energy drinks (ED) consumption and identify its correlation with overweight and obesity.

Setting: Adolescents from 22 high schools in the state of Qatar completed classroomadministered survey.

Participants: 1225 adolescents $(51.2 \%$ boys and $48.8 \%$ girls $)$ in grades $10-12$ aged 15-18 years.

Results: A significant difference between males and females adolescents was reported regarding age $(<0.001)$, body mass index $(\mathrm{P}<0.0001)$ and energy drinks consumption $(\mathrm{P}<0.000)$. Two thirds of adolescents in our sample consumed energy drinks at least once a week. Energy drinks consumption was more prevalent among boys than girls, the consumption energy drink was also significantly related to age and body mass index BMI for both genders $(\mathrm{P}<0.05)$.

Conclusions and Implications: energy drinks consumption could be one of obesity predisposing causes. In addition to its potential health risks primarily because of stimulant content. Future interventions designed to reduce ED consumption to address the clustering of unhealthy habits and behaviors.

Keywords: adolescents, energy drinks, obesity, overweight, body mass index
Research Article

Volume 8 Issue 2 - 2018

\author{
Ghazi Daradkeh,' Asma Al Muhannadi,' \\ Chandra P,2 Moudi Al Hajri' \\ 'Department of Dietetics, AL-Khor Hospital (AKH), Hamad Medical \\ Corporation, Qatar \\ ${ }^{2}$ Department of Medical Research, Hamad Medical Corporation, \\ Qatar
}

Correspondence: Ghazi Daradkeh, Hamad Medical Corporation,AL-Khor Hospital, Qatar,Tel +9745526 47I4, Email GDARADKEH@hamad.qa

Received: February 13, 2018 | Published: March 01, 2018
Abbreviations: ED, energy drinks; BMI, body mass index; IOTF, International obesity task force.

\section{Introduction}

Sports and energy drinks are considered sugar-sweetened, noncarbonated, beverages which contain added sugars, minerals, and electrolytes to help replenish the body during vigorous exercise, but their ingredients and purported functions differ from other sugarsweetened beverages. ${ }^{1}$ High amounts of caffeine often coupled with other natural stimulants that enhance caffeine's effects, are the main ingredients of energy drinks. Increase mental concentration as well as physical performance is purported by beverages marketed of energy drinks. In spite of energy drinks are a source of excess sugar that can increase obesity risk but unfortunately this not well documented. Since young people who may consume these products for non-healthy reasons may derive as much as $12 \%$ of their sugary drink intake from energy drinks ${ }^{2}$ and are found to consume energy drink at high rates on a weekly basis ${ }^{3}$ many may inadvertently be harming themselves in the long run, even if they actively avoid traditional sugary beverages. Adolescent consumption of sugar-sweetened beverages is of concern because consumption is associated with increased risk for dental caries, excess weight gain, and poor diet quality. ${ }^{4-7}$ It was noticed by recent evidence that a decline in the prevalence of soft drink and fruit drink consumption among U.S. adolescents from 1999 to 2008, while sports and energy drink consumption tripled. ${ }^{8}$ In regard to American Academy of Pediatrics (AAP) findings in June 2011, they conclude that there is no benefit provided from energy drink and should not be consumed by adolescents, as these drinks create a risk for overstimulation of the nervous system..$^{9-11}$

Energy drinks among youth become common and popular due to their stimulant qualities. In 2014 a study by Kumar et al. ${ }^{12}$ found that $9 \%$ of youth ages $12-17$ drank energy drinks, almost $20 \%$ felt these was safe for teens. It was noticed that the consumption of energy drinks increased with age among adolescents, and this group misinformed and unaware about the potential health effects and nutritional content of energy drinks. Short sleep durations, obesity and cardio-metabolic diseases associated with obesity are outcomes of consuming energy drinks. ${ }^{12}$ A substantial increase risk for overweight or obesity in children and adolescents as a result of frequent or excessive intake of caloric energy drinks was reported. ${ }^{13}$ To the best of our knowledge, no study in Qatar and in the region has specifically examined the patterns of energy drink consumption among adolescents and its association 
with overweight and obesity. To address this gap in the literature, the present study used data from adolescents-based survey to describe patterns of energy drinks consumption and its relation to weight status.

\section{Methods}

This study aims to investigate the adolescent's energy drinks consumption with body weight status. The design of this study was a cross-sectional survey. Ethical approval by Medical Research Center (MRC) at Hamad Medical Corporation-state of Qatar was obtained. The study was approved by the Ministry of Education in Qatar, as well as the selected schools. All participants consented before participation. This study was conducted in Doha, the capital of Qatar and AL-Khor city-north of Qatar.

\section{Data collection}

The data were collected by using a pre-tested questionnaire. It was used as a base to develop the questions to measure the adolescent's response towards energy drinks consumption. The reliability of the questionnaire was then tested on a group of students before applying the questionnaire to the whole sample. A multistage statistical random-sampling technique was used to select the participants. In the first stage, a systematic random sampling procedure was used to select the schools. The schools were stratified into boys and girls secondary schools, with further stratification into public and private schools. At the second stage, classes were selected at each grade (level) using simple random-sampling design. In this way, one third of sections were randomly selected in each of the three levels 10, 11, 12 from each secondary school. Both boys and girls governmental and private schools were included in the study. Thus, we had a total selection of at least 57 classes ( 29 boys and 28 girls). All students in the selected classes, who were free of any physical health problems, and their age between 15-18 years were selected to participate in the study. In addition, all the schools and students were consented to involve in this study. The total sample size consisted of 1225 adolescents ( 625 boys) and (600 girls).

\section{Anthropometric measurements}

Body weight and height were measured in the morning by a trained researcher according to written standardized procedures. Body weight was measured to the nearest $0.1 \mathrm{~kg}$ using electronic portable scales and height was measured to the nearest $(1.0 \mathrm{~cm})$. Measurements were done with school uniforms and without shoes. Body Mass Index (BMI) was calculated as the ratio of weight $(\mathrm{Kg})$ to height $\left(\mathrm{m}^{2}\right)$.

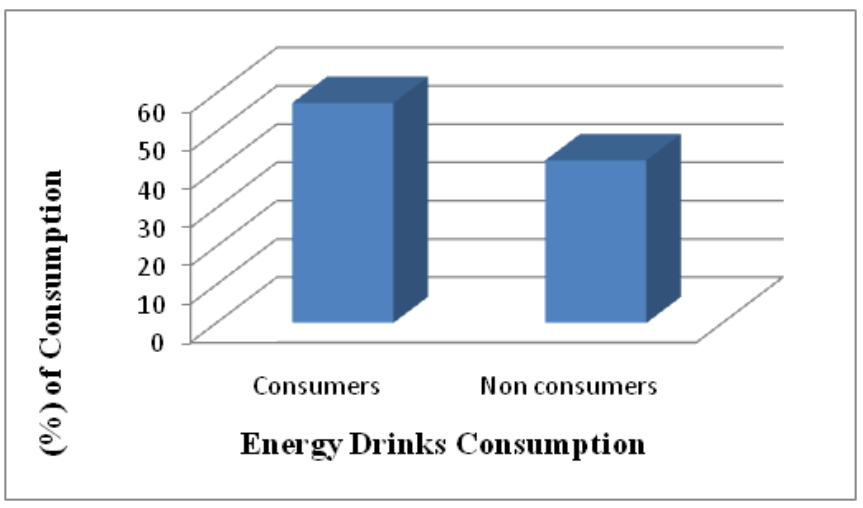

Figure I Overall Prevalence of (ED) Consumptions.
BMI reference values were used to defined overweight and obesity in adolescent aged 14-17 years, based on the classification of the International Obesity Task Force (IOTF) Cole TJ et al. ${ }^{14}$ while adults BMI cut - off points for (overweight $25-29.9 \mathrm{~kg} / \mathrm{m}^{2}$ and obesity $\geq 30$ $\mathrm{kg} / \mathrm{m}^{2}$ ) were used for participants aged 18 years. The adolescents were asked for their consumption of energy drinks as how many times per week the participants consumed. The current study were carried out to investigate the adolescent's intake of energy drinks in Qatar with body weight status. The answers ranging from (never $=0$ intake) to (every day $=7$ time/week). The consumption was classified into two levels of intake: $<4$ times/week, and $\geq 4$ times /week. The data were analyzed using the Statistical Package for Social Sciences software (SPSS, Version 15.0). Questionnaires with missing data were excluded. Description statistics are presented as mean and standard deviation. Chi-square test was used to find the significance between parameters (overweight, obesity, gender, age and frequency of intake). The level of significance was set at $\mathrm{P}<0.05$.

\section{Results and discussion}

The findings of this study and other research indicate energy drink consumption are common among adolescents. ${ }^{15,16}$ In our study, the overall energy drinks were consumed regularly (at least once per week) by $57.5 \%$ of adolescents in our sample Figure 1 . This result similarly to the finding of Nicole et al. ${ }^{15}$ Regular consumption of energy drinks was significantly higher among boys $69 \%$ than among girls $31 \%$ $(P<0.001)$ (Figure 2). This finding is consistent with Nicole Larson et al. ${ }^{15}$ findings; who reported that energy drinks consumption was $44.9 \%$ and $31.6 \%$ among boys and girls respectively. It was noticed that most adolescents, particularly boys, increase their energy drinks as they become older for the most common reasons for use including taste, stimulants to enhance their performance, wakes up, and makes feel alert "It makes me go hyper"and"I drink it before soccer and I don't lose energy as fast. Our study found that regularly consumption of energy drinks was dramatically increased with adolescent's age. The consumption was $18.9 \%, 22.9 \%, 25.9 \%$ and $32.3 \%$ of 15,16 , 17 , and 18 years old adolescents respectively (Figure 3 ). Similarly to Kumar et al. ${ }^{12}$ findings who reported the highest consumption rates of energy drinks was among 16-17 year olds adolescents. Our results showed a significant association between the frequency of energy drinks consumption and weight gains as measured by Body Mass Index (BMI) (Figure 4). Similarly to other reports that obesity was reported as one of the health risks among who exposed to excess carbohydrate contents of these drinks. ${ }^{12,13}$

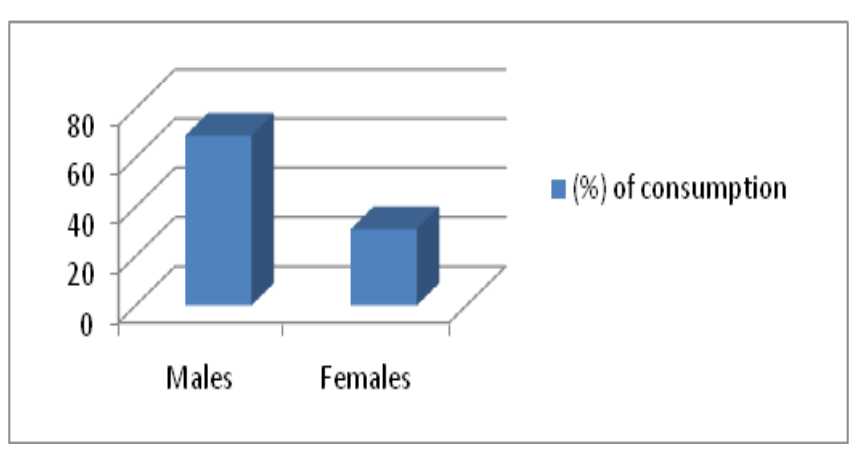

Figure 2 Prevalence of (ED) Consumptions with Sex. 


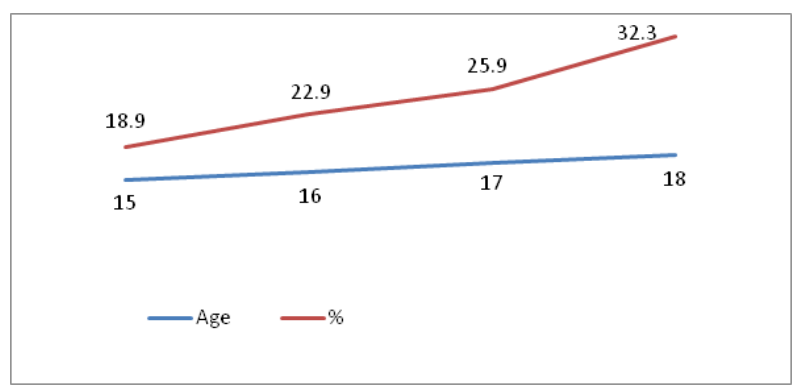

Figure 3 Prevalence of (ED) Consumptions with Adolescent Age.

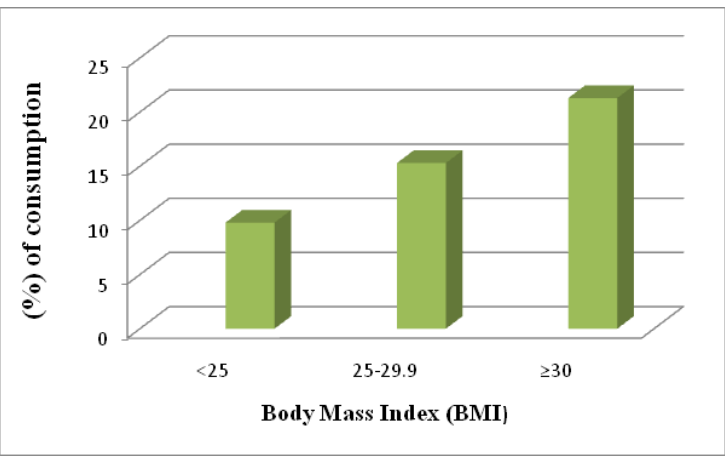

Figure 4 Prevalence of (ED) Consumptions with BMI.

\section{Conclusion}

Adolescent's consumption of energy drinks in Qatar is common, in spite of its content of excess sugar and possible adverse impact in relation to obesity in young adults and adolescents. Encouragement to avoid or limit the use of energy drinks and encourage healthy beverages intake through educational and awareness programs by medical care providers and public health practitioners is an essential need.

\section{Acknowledgments}

We thank the Medical Research Center (MRC) at Hamad Medical Corporation for ethical approval and financial support of this study, as well as, our thanks extended to ministry of education, all schools, all students and all parents for their participation in this study.

\section{Conflicts of interest}

None.

\section{References}

1. Galemore C. Sports drinks and energy drinks for children and adolescentsare they appropriate? A summary of the clinical report. NASN Sch Nurse. 2011;26(5):320-321.
2. Gómez-Miranda LM, Bacardí-Gascón M, Caravalí-Meza NY, et al. Consumption of sweetened, energy and alcoholic be $\neg$ verages among college students in the México-US border. Nutr Hosp. 2014;31(1):191195.

3. Styer AM, Roesch SL, Argyropoulos G. Modulation of fibroblast growth factor 19 expression by bile acids, meal replacement and en $\neg$ ergy drinks, milk, and coffee. PloS One. 2014;9(1):e85558.

4. Malik V, Popkin B, Bray G, et al. Sugar-sweetened beverages, obesity, type 2 diabetes mellitus, and cardiovascular disease risk. Circulation. 2010;121(11):1356-1364.

5. Ebbeling C, Feldman H, Chomitz V, et al. A randomized trial of sugarsweetened beverages and adolescent body weight. $N$ Engl J Med. 2012;367(15):1407-1416.

6. Berkey CS, Rockett HR, Field AE, et al. Sugar-added Beverages and adolescent weight change. Obes Res. 2004;12(5):778-788.

7. Shenkin J, Heller K, Warren J, et al. Soft drink consumption and caries Risk in children and adolescents. Gen Dent. 2003;51(1):30-36.

8. Han E, Powell L. Consumption patterns of sugar-sweetened beverages in the United States. J Acad Nutr Diet. 2013;113(1):43-53.

9. Rath M. Energy drinks: what is all the hype? The dangers of energy drink Consumption. J Am Acad Nurse Pract. 2012;24(2):70-76.

10. Reissig C, Strain E, Griffiths R. Caffeinated energy drinks a growing problem. Drug Alcohol Depend. 2009;99(1-3):1-10.

11. Seifert S, Schaechter J, Hershorin E, et al. Health effects of energy drinks on children, adolescents, and young adults. Pediatrics. 2011;127(3):511528.

12. Kumar G, Park S, Onufrak S. Perceptions about energy drinks are associated with energy drink intake among US youth. Am J Health Promot. 2015;29(4):238-244.

13. Committee on Nutrition and the Council on Sports Medicine and Fitness. Sports drinks and energy drinks for children and adolescents. Are they appropriate? Pediatr. 2011;127(6):1182-1189.

14. Cole TJ, Bellizzi MC, Flegal KM, et al. Establishing a standard definition for child overweight and obesity worldwide: International survey. BMJ. 2000;320(7244):1240-1246.

15. Nicole Larson, Jessica DeWolfe, Mary Story, et al. Adolescent Consumption of Sports and Energy Drinks: Linkages to Higher Physical Activity, Unhealthy Beverage Patterns, Cigarette Smoking, and Screen Media Use. Nutr Educ Behav. 2014;46(3):181-187.

16. O'DeaJA. Consumption of nutritional supplements among adolescents: Usage and perceived benefits. Health Educ Res. 2003;18(1):98-107.

17. Cinteza E. Update in pediatrics: to take or not to take soft drinks, sports or energy drinks. Maedica. 2011;6(2):157-158. 